\title{
The Role of Zinc Oxide Nanoparticles in Sesamum Indicum Growth and Yield
}

\author{
Narendhran $\mathrm{S}^{1,2 *}$, Rajiv $\mathrm{P}^{2}$, Rajeshwari Sivaraj ${ }^{3}$, Brindha $\mathrm{P}^{1}$ and Cruz Charles ${ }^{1}$ \\ ${ }^{1}$ Department of Biotechnology, India. \\ ${ }^{2}$ Department of Biotechnology, , India \\ ${ }^{3}$ Department of Chemistry, , India
}

*Corresponding author: Narendhran S, Department of Biotechnology, Sri Ramakrishna College of Arts and Science College, India

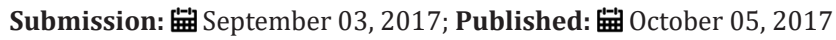

\begin{abstract}
As of late fast progressed in comprehension synthesis and engineering nanoparticles without a doubt will proceed with disturbing development of products enveloped with nanomaterials. The green synthesis method has prompted the improvement of biomimetic methodologies for the development of advanced nanomaterials. Biological methods for nanoparticles synthesis utilizing plants separate have been recommended as could reasonably be expected eco-friendly different option for chemical and physical methods. Lantana aculeate has many negative impacts including potential to disrupt succession cycle, displacing native biota resulting in decreased biodiversity and noteworthy issue in agricultural areas. This plant can be used for the nanoparticles synthesis in eco-friendly manner and also due to its rapid propagation. The study reports the production of zinc oxide nanoparticles from L. aculeate, zinc nitrate and assessing it plant growth attribute of Sesamum Indicum. The biogenic Zinc Oxide nanoparticles (ZnO NPs) have promising antifungal activity, germinating at lower concentration and significant contribution to level of yield in crop cultivation when compared to zinc nitrate mediated ZnO NPs and commercial available NPs. Gene expression provides an early warning of stressful effects. The fold change of superoxide dismutase has comparatively high than glutathione reductase and catalase.
\end{abstract}

Keywords: Zinc Oxide nanoparticles, Lantana aculeate, Sesamum Indicum, Plant growth attribute

Abbreviations: Bulk ZnO: Commercially Available Zinc Oxide Nanoparticles; CAT: Catalase; Chemical ZnO: Zinc Nitrate Mediated Zinc Oxide Nanoparticles; gL ${ }^{-1}$ : Gram Per Liter; GR: Glutathione Reductase; Green ZnO: L. Aculeate Mediated Of Zinc Oxide Nanoparticles; nm: Nanometer; NPs: Nanoparticles; SOD: Superoxide Dismutase; ZnO: Zinc Oxide Nanoparticles

\section{Introduction}

A simple and rapid procedure has been developed to synthesize ZnO nanoparticles using Lantana aculeate leaf extract and zinc nitrate. The synthesized nanoparticles were characterized by UVVis spectrophotometric, Fourier transform infrared spectrometer, X-ray diffractometer, Energy dispersive X-ray spectrometer, Field emission scanning electron microscopy, High resolution transmission electron microscopy and particle size analysis. The ZnO nanoparticles synthesized from biological and chemical method showed particles are spherical shape with size range from $12-25 \mathrm{~nm}$ and $18-35 \mathrm{~nm}$ respectively [1,2]. Biological synthesis of nanoparticles showed particles with distinct cap, which may be due to flavonoids, proteins and other functional group present in the leaf broth of Lantana aculeate.

Antifungal activities of synthesized $\mathrm{ZnO}$ nanoparticles on plant fungal pathogens were studied [1]. From the outcomes, it was found that the antifungal impact of synthesized zinc oxide nanoparticles has been attributed to their size and high surface to volume ratio. The green synthesized $\mathrm{ZnO}$ nanoparticles inhibited fungal organism in the following order:
Aspergillusniger(MTCC:10180)>Fusariumoxysporum (MTCC: 3326)>Fusariumoxysporum (MTCC: 3327)>Fusariumoxysporum (MTCC: 3930)>Penicillianfuniculosum (MTCC: 4888)

Whereas chemical synthesized ZnO nanoparticles inhibition in the order of Fusariumoxysporum(MTCC: 3326)>Aspergillus niger (MTCC: 10180)>Penicillianfuniculosum (MTCC: 4888)>Fusariumoxysporum (MTCC: 3930)>Fusariumoxysporum (MTCC: 3327).

However, bulk $\mathrm{ZnO}$ nanoparticles showed inhibitory activity in the order of Fusariumoxysporum (MTCC: 3326)>Fusariumoxysporum (MTCC: 3327)>Fusariumoxysporum (MTCC: 3930)>Penicillianfuniculosum (MTCC: 4888) >Aspergillus niger (MTCC: 10180).

From the morphological, biometric and yield parameter of Sesamum Indicum plant (Variety C0-1) [7], Green ZnO showed less toxic effects when compared with zinc sulphate followed by chemical ZnO nanoparticles. Total zinc accumulated in plant sample were found to be in order of Green $\mathrm{ZnO}>$ bulk $\mathrm{ZnO}>$ chemical $\mathrm{ZnO}$.

Lantana aculeate mediated zinc oxide nanoparticles at $0.5 \mathrm{gL}^{-1}$ concentration increased the growth attributes, quality parameters 
and yield components of Sesamum Indicum than the control. When the concentration of nanoparticles increase then the growth and yield component were decreased. The green synthesized $\mathrm{ZnO}$ and bulk ZnO nanoparticles treated Sesamum Indicum shows increased in DNA damage at $1000 \mathrm{mg} / \mathrm{L}$ concentration but chemical synthesized $\mathrm{ZnO}$ observed in $0.25 \mathrm{gL}^{-1}$ concentration. Chemical synthesized $\mathrm{ZnO}$ nanoparticles showed increased activity of ROS generated due to nanoparticles stress followed by bulk $\mathrm{ZnO}$ and then by green $\mathrm{ZnO}$.

In the present experiment, excess metal toxicity increased the activity of SOD (Superoxide dismutase), GR (Glutathione reductase) and CAT (Catalase). It may be concluded that appropriate amount of metal could reduce the damage of active oxygen under metal stress, but reversely excessive level of metal (Zn) were just to aggravate the already serious damage to the plants. $\mathrm{Zn}$ is a possible environment toxicant for plant under excessive conditions. To understand the molecular stress response involved in exposure of bulk and nano form of chemical and green synthesized ZnO. We focused on the transcript accumulation of three stress-related genes: SOD, GR and CAT. Transcript accumulation of the above mentioned stress related gene were determined on nanoparticle stressed Sesamum Indicum plant by using a quantitative real-time PCR (qRT-PCR) analysis.

The plants were exposed to bulk, biological and chemical $\mathrm{ZnO}$ of $0.1-2 \mathrm{gL}^{-1}$ and $\mathrm{ZnSO}_{4}$ of $0.25 \mathrm{gL}^{-1}$. The treated plants were not affected morphologically by bulk and biological ZnO NPs but higher concentration of chemical $\mathrm{ZnO}$ showed differences in the plant morphology like bending of plant, growth inhibition, curling of leaves and so on. However, the plant treated with chemical synthesized ZnO NPs showed several toxicities at lower concentrations. The result revealed that up-regulation reduced, showing that higher concentrations of chemical synthesized ZnO NPs may cause destabilization of the cellular homeostasis, implying that at these levels, the plants cannot react to stress due to deterioration. However, the case is compromising for green synthesized ZnO NPs were disruption was noticed at a concentration above $1 \mathrm{gL}-1$. So for the current study whole plant treated with only lower concentration of bulk, biological and chemical ZnO NPs were selected for transcript accumulation studies.

The genes were up-regulated for bulk and $\mathrm{ZnSO}_{4}$ stressed sesame but whereas in case of chemical and green synthesized $\mathrm{ZnO}$ nanoparticles gene got over expressed at lower concentration and later down regulation was noticed. It seems that the plant does not react to NP stress due to the destabilization of homeostasis. Transcript level were normalized with housekeeping gene Act in and compared with the control group. The highest relative fold change was measured on SOD, GR and CCS gene. It was noted that the plant sample treated with CZnO showed maximum fold change of 7.653 for SOD, 1.186 for GR and 0.932 for CCS. It has been demonstrated that the qualities in charge of cell metal homeostasis are directed by different substantial metals at the transcription level $[3,4]$.

In case of green $\mathrm{ZnO}$ treated plant samples, fold change of 6.395 for SOD, 1.053 for GR and 0.357 for CCS was noticed. However, bulk $\mathrm{ZnO}$ and $\mathrm{ZnSO}_{4}$ treated plants showed low level of fold change when compared to chemical and green synthesized ZnO NPs. GR play a crucial role in determining the tolerance of a plant under various type of stress [5] and recently it has been observed that the GR activity increases in the nearness of $\mathrm{Cd}$ in different plants. GR has showed also differential responses under As (V) and Cr (VI) stress. Because Hsp90-1, MT2 and GR1 like protein, transcripts all accumulated stress, a combinatorial kind of resilience instrument identified with protein damage repair, metal chelation and the antioxidative metabolism could be effectively activated in sesame plants to provide protection against toxicity [6].

Liu et al. [7] reported that particularly under hypoxia stress, excess Mn increased the activities of SOD, APX and GR. Over expression of certain antioxidant gene such as SOD, APX and CAT protect from an assortment of anxieties in which the quality items diminishes the cell damage by scavenging the ROS. However, the activation of these genes could be a protection mechanism to metal and nanoparticle stress, other than the transporter system. Numerous isoforms of relating genes are accessible in the database. So more studies would give profitable results to screen which isoform would demonstrate productive and assume imperative part in the detoxification or resilience component.

\section{Discussion}

Synthesized zinc oxide nanoparticles showed promising antifungal activity against Aspergillus niger, Fusarium oxysporum and Penicillium funiculosum. Various concentrations of green $\mathrm{ZnO}$, bulk $\mathrm{ZnO} \&$ chemical ZnO nanoparticles effect on germination and root elongation of Sesamum indicum were studied. Metal oxide are quickly transported through the plant and included in the metabolic processes through soaking methods. It's prove that Sesamum Indicum seeds germination at lowest concentration $\left(0.1 \mathrm{gL}^{-1}\right)$ of green $\mathrm{ZnO}$ suspension solution proved good root growth compared to bulk $\mathrm{ZnO}$ and chemical ZnO NPs. Expanding centralization of green $\mathrm{ZnO}$ nanoparticles altogether lessened the development of plant. It may involve agronomic traits to control the weed seed germination.

Zinc accumulation in plant samples were found to be high in order of green $\mathrm{ZnO}>$ bulk $\mathrm{ZnO}>$ chemical $\mathrm{ZnO}$ nanoparticles treatment. The results of these studies indicate that biologically synthesized ZnO nanoparticles showed less toxic effects when compared with chemically synthesized ZnO nanoparticles. Larger amount of zinc was existent in the nanoparticles treated seeds and which was reasonable for development in zinc inadequate soil and that enhanced the grain yield. Application of zinc oxide nanoparticles can make a significant contribution to level of yield in crop cultivated in zinc deficient soil.

The percentage of tail DNA in Sesamum Indicum treated with different concentration of zinc oxide nanoparticles. While green $\mathrm{ZnO}$ nanoparticles showed a signs of significant DNA damage at higher concentration $\left(1 \mathrm{gL}^{-1}\right)$, bulk $\mathrm{ZnO}$ particles induced a dose depended increase in extent of DNA damage with significantly at concentration above $0.5 \mathrm{gL}-1$. But chemical $\mathrm{ZnO}$ nanoparticles increase in DNA damage at lower concentration $\left(0.25 \mathrm{gL}^{-1}\right)$. This could be credited to a property of nanomaterials to frame 
agglomerates by goodness of which, with expansion in treatment focus the nanoparticles tend to precipitate $[8,9]$.

Genotoxicity of $\mathrm{ZnO}$ nanoparticles was assessed on Sesamum indicum by comet assay and further confirmed by DNA laddering. A greater amount shearing of DNA was noted in chemical $\mathrm{ZnO}$ nanoparticle treatment when compared to green $\mathrm{ZnO}$ nanoparticles. Gene expression provides an early warning of stressful effects. The fold change of superoxide dismutase has comparatively high than glutathione reductase and catalase.

\section{Conclusion}

Biological synthesized zinc oxide nanoparticles have good growth and yield of Sesamum Indicum when compared to chemical and bulk nanoparticles. They help to antifungal agent and increase the crop yield considerably. Lantana aculeate can be used for production of nanoparticles effectively.

\section{References}

1. Narendhran S, Rajeshwari S (2015) Biogenic ZnO nanoparticles synthesized using Lantana aculeata leaf extract and their antifungal activity against plant fungal pathogens. Bull Mater Sci 39(1): 1-5.

2. Narendhran S, Rajiv P, Rajeshwari S (2015) Toxicity of ZnO nanoparticles on germination of Sesamum Indicum (Co-1)and their antibacterial activity. Bull Mater Sci 39(2): 415-421.
3. Bennett DR, Baird CJ, Chan KM (1997) Zinc toxicity following massive coin ingestion. Am J Forensic Med Path 18(2): 148-153.

4. Connolly EL, Fett JP, Guerinot ML (2002) Expression of the IRT1 metal transporter is controlled by metals at the level of transcript and protein accumulation. Plant Cell 14(6): 1347-1357.

5. Narendhran S, Rajiv P, Rajeshwari S (2016) Influence of zinc oxide nanoparticles on growth of Sesamum Indicum L. in zinc deficient soil. Int J Pharm Pharm Sci 8(3): 365-371.

6. Gill SS, Tuteja N (2010) Reactive oxygen species and antioxidant machinery in abiotic stress tolerance in crop plants. Plant Physiol Biochem 48(12): 909-930.

7. Liu A, Chen S, Mi Y, Zhou Z, Ahammed G (2010) Effect of hypoxia stress and different level of $\mathrm{Mn}^{2+}$ on antioxidant enzyme of tomato seedling. Am J Plant Sci 1(1): 24-31.

8. Rao ASVC, Reddy AR (2008) Gluthathione reductase: A putative redox regulatory system in plant cells. In: Khan NA, Singh S, Umar S (Eds.), Sulfur assimilation and abiotic stresses in plants. Berlin Heidelberg: Springer-Verlag, pp. 111-147.

9. Zhu H, Han J, Xiao jQ Jin Y (2008) Uptake, translocation and accumulation of manufactured iron oxide nanoparticles by pumpkin plants. J Environ Monit 10(6): 713-717. 